\title{
CLASSICAL VARIATIONAL BASIS FOR SCHRÖDINGER QUANTUM THEORY*
}

\author{
James G. Gilson \\ School of Mathematical Sciences \\ Queen Mary College, Mile End Road \\ London E1 4NS, United Kingdom
}

\begin{abstract}
A two-dimensional classical hyperspace fluid theory of the vacuum which is an alternative to Schrödinger quantum theory involves a "stirring" energy density. This energy density, like entropy density, measures the local "disturbance" involved by the time evolution of a state of the system. The stirring energy density is shown to be the local source term for the rate of generation of action density with time. A classical fluid action principle is solved using the "action" of this source term without assuming quantum time evolution. Schrödinger quantum theory is recovered from this new action principle which is then discussed. Finally, the quantity made stationary by the variational principle measures disturbance in the environment as an angular change in the orientation of the whole system.
\end{abstract}

Key words: Schrödinger, Quantum, Classical, Fluids, Vorticity

AMS Subject Classification: 81C05, 76C05

\section{INTRODUCTION}

This paper arises from the author's work [1,2] aimed at producing a fluid basis for Schrödinger quantum theory which is classically explicable. This work also involves the objective of using physically significant arguments, within a mathematical scheme of maximal simplicity, in order to give a deeper understanding of the fundamental processes that underlie the Schrödinger equation type of description of physical events. This later objective is the main motivation for the following discussion. More detailed information about the basis and structure of the alternative view of Schrödinger quantum theory used in

\footnotetext{
* Received June 3, 1988; Revised and Accepted, November 3, 1988.
} 
this article can be found in references $[3,4,5]$. An early fluid interpretation for quantum theory was given by Madelung [16]. Related approaches to the problems of reformulating quantum theory can be found in references [17, 18, 19, 23, 24]; and a wide-ranging discussion of the various points of view with many references can be found in Max Jammer's book [20]. Recent analysis of some philosophical issues has been given by Peres [21]; and great progress involving much clarification of the fundamental issues involved in reformulating Schrödinger theory is to be found in the recent works by Cavalleri and Spavieri $[25,26]$ on the subject of zitterbewegungen. From the electromagnetic point of view, the theoretical structure to be used in this article has some relationship with magnetohydrodynamics [7].

In the Gilson reference [1], it was demonstrated that it is possible to deduce Schrödinger quantum theory from a "classical" variational principle very much like the variational principles used in the theory of superfluids and superconductivity $[6,13,14]$. This was the basis of the author's suggestion that the concept of a "superfluid" is possibly a more fundamental physical idea or mathematical model for some aspects of natural phenomena than is the orthodox Schrödinger equation model, thus making the superfliud a basic, primitive, underlying, physical structure. Subsequent study has revealed that there is a related and much simpler variational principle which leads from classical fluid theory to quantum mechanics, with the additional advantage that its "physical" significance is of substantially greater clarity. The derivation of quantum mechanics from this new variational principle constitutes the main concern of this paper.

\section{ACTION DENSITY}

In the Gilson reference [2], it was shown that the "classical" fluid complex potential has special significance in Schrödinger quantum theory. When we are working with the Schrödinger equation, this complex potential exists in the two-dimensional hyperspace associated with the analytic continuation of a configuration coordinate. The velocity field that it controls can be regarded as representing the relative motion of mass "monopoles" existing in the hyperspace. Let us denote its real and imaginary parts by a pair of conjugate harmonic functions, $\alpha_{1}(x, y, t)$ and $\alpha_{2}(x, y, t)$. These functions can both be regarded as carrying "information": $\alpha_{2}$ is directly related to entropy or missing information, while $\alpha_{1}$ is directly related to the classical time action integral of a Lagrangian function $L$ [2]. The use of a Lagrangian function in the form of an action "density" in classical variational principles 
is well-established [6, 4], especially in fluid theory. However, the physical significance of an action density is not very clear. On the other hand, the physical or statistical significance of an entropy "density" is rather clear. In both of these cases, either entropy or action is often treated and manipulated conceptually and mathematically as though it were a measure of quantity of "material" substance, as is mass density for example. The use of action in this way means that it must have an "additive" property that, from the point of view of the present work, can be expressed as follows. If the action per dipole is $\alpha_{1}$ and there are $\rho^{(0)}$ dipoles in a small volume $v_{0}$, then the action in the volume $v_{0}$ due to dipoles is $\alpha_{1} \rho^{(0)}$. The two components of the complex potential in the quantum fluid context are consequently in this "slightly" uncertain characterization role, a point of significance that does require more analysis. However, with this reservation in mind, we shall make use of the idea of action density to arrive at some very interesting conclusions.

Let us assume that a complex potential $\alpha=\alpha_{1}+i \alpha_{2}$ for the relative monopolar motion exists. If $m_{0}$ is the electronic rest mass, $m_{0} \alpha_{1}$ has the dimensions of action and relates directly to the classical dynamical action for a particle of rest mass $m_{0}$. Suppose that we also have information about the local density of dipoles formed from pairs of "opposite" mass monopoles given in terms of a number density function $\rho(x, y, t)$. We shall take $\rho$ to be a density per unit three-space volume. It is convenient to work in a three-space for a number of reasons. The two coordinates $x$ and $y$ refer to a cross section of the three-space while all the primary dipoles are regarded as pointing in the third direction orthogonal to the $x-y$ hyperspace. The third direction also enables the use of vector representations for the vorticity of the $x-y$ flow and for other dynamical and electrical characteristics [5] which are involved. Thus if $m_{0} \alpha_{1}$ is the action per dipole, then the local dipole action density per unit volume is given by

$$
\sigma^{(\mathrm{act})}=m_{0} \alpha_{1}(x, y, t) \rho(x, y, t) .
$$

The velocity field for the dipolar motion $\beta^{(c)}$ is the centroidal velocity of the monopolar velocity fields,

$$
\beta^{(c)}=(1 / 2)\left[u(x, y, t) e+v(x, y, t) e^{\prime}\right]
$$

The equation of continuity expressing conservation of the dipole number and taking into account the possibility of new local dipole formation by the external field is given by 


$$
\frac{\partial \rho}{\partial t}+\nabla \cdot\left(\rho \beta^{(c)}\right)=\Gamma
$$

where $\Gamma$ is the source function and is of the form

$$
\Gamma=\rho V_{2} / m_{0} v
$$

with

$$
v=c l_{0}=h / 2 m_{0} .
$$

The quantity $v$ will be used as a "fundamental" amount of action. It is equivalent to the magnetic diffusivity in magnetohydrodynamics [7]. $V_{2}$ is the imaginary part of the analytically continued external potential, $V_{1}(x)$, and $c$ is the velocity of light. Thus $V_{2} / m_{0} v$ gives the time rate of production of density per unit density present. We can find the local source term, $\Gamma^{(\text {act })}$, for the time rate of production of action in terms of the quantities introduced as follows. The appropriate equation of continuity for the conservation of action will be

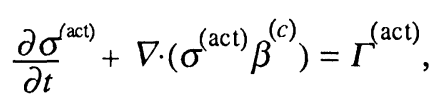

if we assume that the transported action per dipole is carried by the dipolar centroidal velocity field (2.2). Thus using (2.3), we find

$$
\Gamma^{(\mathrm{act})}=\rho m_{0} \frac{\partial \alpha_{1}}{\partial t}+\rho\left(m_{0} \nabla \alpha_{1}\right) \cdot \beta^{(c)}+m_{0} \alpha_{1} \Gamma
$$

We are assuming that $\alpha_{1}$ is the velocity potential for the relative monopolar motion,

$$
\bar{\beta}=u(x, y, t) e-v(x, y, t) e^{\prime} .
$$

Consequently,

$$
u=-\frac{\partial \alpha_{1}}{\partial x}
$$


and

$$
-v=-\frac{\partial \alpha_{1}}{\partial y}
$$

If we assume for the moment that we are dealing with a quantum system, then the Schrödinger local energy can be identified with the well-known time derivative of the velocity potential that occurs in the Bernoulli equation for the flow [11]. That is

$$
E_{Q} / m_{0}=\frac{\partial \alpha_{1}}{\partial t}
$$

The appropriate Bernoulli equation itself has the form

$$
E_{Q}=\left(m_{0} / 2\right)\left(u^{2}-v^{2}\right)+\left(m_{0} v / 2\right)(\partial v / \partial x-\partial u / \partial y)+V_{1} .
$$

Thus when we use the last four equations, (2.7) reduces to

$$
\begin{aligned}
\Gamma^{(\text {act })} & =\left(\left(m_{0} v / 2\right)(\partial v / \partial x-\partial u / \partial y)+V_{1}+\alpha_{1} V_{2} / v\right) \rho \\
& =E^{\text {(stir) }} \rho(x, y, t) .
\end{aligned}
$$

So that, under quantum conditions, the "local" time rate of production of action is the "energy" density on the right-hand side of (2.13). In (2.13), there are three distinct and clearly recognizable contributions to the local production of action density. The first term is a contribution from the vorticity of the monopolar flow over the $x-y$ plane. The second term is simply the contribution from the external field, while the third term is action associated with the new "density" resulting from the source term in the continuity equation for local density production.

It is tempting to regard the energy density (2.13) as a measure of the "stirring up" of the polarizable vacuum resulting from the time evolution of the system, in much the same way as entropy measures disorder in a system. Such a view would seem to be consistent with the idea that the action density transported from place to place by the dipolar centroidal velocity field according to the continuity equation (2.6) is more closely associated with the ordered motion of the system. It will be shown that we can deduce the Schrödinger equation by means of a variational principle using the "stirring" energy density (2.14) as the integrand in a Lagrangian action integral. Consider the variational principle defined by 


$$
\delta \int_{t_{0}}^{t} \iint_{S}\left(\Gamma^{(\mathrm{act})}+\lambda\left(\partial \rho / \partial t^{\prime}+\nabla \cdot\left(\rho \beta^{(c)}\right)-\rho V_{2} / m_{0} v\right) m_{0}\right) d x d y d t^{\prime}=0
$$

The $S$ by the integral sign means an integral over a region of "area" $S$ of a two-dimensional $x-y$ hyperspace. For future reference, let us denote the quantity that is being varied by $L^{\text {(syst). }}$

$$
L^{\text {(syst) }}=\int_{t_{0}}^{t} \iint_{S} \Gamma^{(\text {act) }} d x d y d t
$$

In (2.15), $\lambda$ is a Lagrange multiplier and the constraint is the conservation equation for dipole density with the source term (2.4). $\Gamma^{\text {(act) }}$ is to be expressed in terms of $u, v, \rho, V_{1}$, $V_{2}$ and $\alpha_{1}$, the velocity potential for the relative monopolar velocity field $\bar{\beta}$, as in (2.9) and (2.10). The constraint term in equation (2.15) involves minor technical improvement in that it now involves the source term for density, $\rho V_{2} / m_{0}$. This was not present in the variational principle as expressed in reference [1]. Working from the expression (2.15), we now do "not" assume that (2.12) holds, but rather we hope that the variational principle will generate this special relation, and so indicate that the system is exhibiting quantum time evolution behaviour. Thus we are asking how the system should move, how the local energy should be related to the local velocity field, and how local velocity should be related to local density in order that the "stirred" up action over some region of space and time shall be stationary or minimal. The answer to this question is easily obtained from the three Euler-Lagrange equations obtained by using (2.15) and taking $u, v$, and $\rho$ to be three independent variables. The three equations are

$$
\begin{aligned}
& \frac{\partial \lambda}{\partial x}-v \frac{\partial \ln \rho}{\partial y}=0, \\
& \frac{\partial \lambda}{\partial y}+v \frac{\partial \ln \rho}{\partial x}=0,
\end{aligned}
$$

and 


$$
\frac{\partial \lambda}{\partial t}+\frac{u}{2} \frac{\partial \lambda}{\partial x}+\frac{v}{2} \frac{\partial \lambda}{\partial y}-\frac{v}{2}\left(\frac{\partial v}{\partial x}-\frac{\partial u}{\partial y}\right)-\frac{V_{1}}{m_{0}}-\frac{\alpha_{1} V_{2}}{v m_{0}}+\frac{\lambda V_{2}}{v m_{0}}=0
$$

As we are assuming that $\alpha_{1}$ is the velocity potential for the relative monopolar motion, (2.9) and (2.10) are valid. As we want (2.19) to reduce to (2.12) and there is no obvious reason why the imaginary part of the external potential should appear in the "real" part of the energy equation, an obvious expedient is to take

$$
\lambda=\alpha_{1}
$$

This eliminates the last two terms from (2.19). From (2.17) and (2.18), we also get

$$
u=-\partial \lambda / \partial x=-v \partial \ln \rho / \partial y
$$

and

$$
v=\partial \lambda / \partial y=-v \partial \ln \rho / \partial x
$$

Further, (2.19) can be put into the form

$$
\partial \alpha_{1} / \partial t-\left(u^{2}-v^{2}\right) / 2-(v / 2)(\partial v / \partial x-\partial u / \partial y)-V_{1} / m_{0}=0
$$

and with (2.11) we have recovered (2.12), the Bernoulli equation that is equivalent to the Schrödinger equation. (2.21) and (2.22) enable us to identify the stream function for the relative monopolar motion as

$$
\alpha_{2}=v \ln \rho(x, y, t)
$$

because (2.21) and (2.22) are the Cauchy-Riemann equations for the real and imaginary parts of the complex potential $\alpha$. The functional shape of $\alpha_{2}$ suggests that, apart from a numerical factor, it can also play the part of an entropy per unit fluid element. Thus the variational principle (2.15) leads to the fluid hyperspace version of Schrödinger quantum theory, with a form very similar to the two-fluid theory for superfluids $[6,13,14]$. In the 
next section, we shall examine another interpretation for the real part of the complex potential $\alpha$ which throws light on its significance in the quantum type fluid situation.

\section{MONOPOLE ORIENTATION}

In the Gilson reference [2], it was shown that the hyperspace fluid version for Schrödinger quantum theory could be expressed in a very simple form in terms of local rotation rates or angular velocities in the hyperspace. The statement,

$$
\Omega_{r}=\Omega_{\mathrm{ex}},
$$

represents a very compact form for Schrödinger quantum theory, with a remarkably clear physico-geometrical meaning. In (3.1), $\Omega_{r}$ is a local angular velocity of monopolar pairs following the dipolar centroidal velocity field, "relative" to the local angular velocity of the monopolar flow. These quantities are given in more detail by equations (3.2) and (3.3).

$$
\Omega_{\mathrm{ex}}(x, y, t)=V_{1} k / m_{0} c l_{0},
$$

where $V_{1}$ is the usual external field term in the Schrödinger equation and $k=e^{\wedge} e^{\prime}$ is a constant unit vector orthogonal to the $x-y$ hyperspace.

$$
\Omega_{r}=d\left(\alpha_{1} k / c l_{0}\right) /\left.d t\right|_{\beta^{(s)}}-\nabla^{\wedge}\left(\beta^{(c)}\right)
$$

The second term on the right-hand side of (3.3) is the vorticity angular velocity of the monopolar flow. We can use this view of the quantum process to help explain the role of the "stirring" energy and action of the last sections.

In this fluid description of the hyperspace vacuum, "opposite" pairs of mass monopoles form "instantaneous" dipoles. The velocity field for the monopolar motion is given by

$$
\beta=u(x, y, t) e+v(x, y, t) e^{\prime} .
$$

The motion of the mass dipoles is usually best described by the "centroidal" velocity field of the "monopoles" (2.2). However, we shall refer to $\beta^{(c)}$ as the dipolar centroidal field. 
In the general situation, the velocity field of the monopolar flow (3.4) will have a nonzero "local" rotation rate arising from its vorticity $\zeta$. This is best regarded as a "collective" rotation rate determined by the relative rotatory motion of fluid "elements," and we shall denote it by

$$
\tilde{\Omega}(x, y, t)=(1 / 2) \zeta=(1 / 2) \nabla^{\wedge} \beta .
$$

We shall assume that the vacuum fluid can also execute a local "intrinsic" type of rotation, relative to the collective rotation given by (3.5), which can either come from the external field through (3.2) or be a consequence of "feedback" from inside the system. This later aspect is important in the case of nonlinear Schrödinger systems $[8,9,10,27]$, which can also be represented by the present formalism with a minor adjustment in the way the energy of the system is defined. Formula (3.3) suggests that it should be possible to give a complete specification of the change of state of the system as a function of space and time, in terms of the local moving changes of "angular" orientation of monopole pairs over the time interval involved. It should be noted that the local rotation rate of the dipole centroidal motion is "half" the local rotation rate of the monopolar velocity field because of the half factor between (2.2) and (3.4). Thus

$$
\nabla^{\wedge} \beta^{(c)}=(1 / 2) \nabla^{\wedge} \beta=\tilde{\Omega}
$$

Formula (3.3) involves following the dipolar centroidal field while measuring the monopolar pair rotation rate. This presents no problem provided we keep in mind that, if we wish to describe the actual rotation rate of the dipolar centroidal velocity field following the dipolar centroidal flow, then equation (3.3) should be divided through by 2 to take into account (3.6).

We are thus motivated to define the "external"-field-induced "relative" monopolar rotation, $\Delta \varphi_{\mathrm{ex}}$, along the path $r^{(c)}(t)$ defined by the moving dipole centroids,

$$
d r^{(c)} / d t=\beta^{(c)}
$$

between times $t_{0}$ and $t$, as 


$$
\Delta \varphi_{\mathrm{ex}}\left(t, t_{0}\right)=\int_{t_{0}}^{t} \Omega_{\mathrm{ex}}\left(r^{(c)}\left(t^{\prime}\right), t^{\prime}\right) d t^{\prime}
$$

The angle given by (3.8) is "relative" in two senses. It is a "change" of angular orientation taking place between the times $t_{0}$ and $t$ and so is relative to whatever the angle is at time $t_{0}$. It is also "relative" to whatever rotation the "natural" frames undergo along the same path and between the same times. This later relativity is implied by equations (3.1) and (3.3). The natural frames rotate with the local angular velocity of the monopolar flow (3.5). Let us now consider the change in orientation of the monopolar pairs between times $t$ and $t_{0}$ relative to the hyperspace rather than relative to the natural frames. Suppose this is given by the angle $\Delta \varphi$. Then

$$
\Delta \varphi=(1 / v) \int_{t_{0}}^{t}\left(d \alpha_{1}\left(r^{(c)}\left(t^{\prime}\right), t^{\prime}\right) / d t^{\prime}\right) d t^{\prime}
$$

The integrand here is the first term in (3.3) and this term has been taken to have a form which gives a "total" differential in (3.9). It will be shown that the assumption of this form for the integrand in defining the orientation angle $\Delta \varphi$ also leads to the conclusion that a system with the structure implied by (3.1), (3.2) and (3.3) will necessarily exhibit quantum behaviour.

The obvious consequence of the definition (3.9) is that, upon integrating between the times $t_{0}$ and $t, \Delta \varphi$ assumes the form of the difference of two terris, one referring to the time $t_{0}$ and the other referring to the time $t$ :

$$
\Delta \varphi=\varphi(x(t), y(t), t)-\varphi\left(x\left(t_{0}\right), y\left(t_{0}\right), t_{0}\right) .
$$

Thus, the situation is reminiscent of the way entropy can be introduced into classical thermodynamics [12]. The total differential implies that a function $\varphi$ exists that is actually "characteristic" of the state of the system at each specific moment of time. In fact, $\varphi(x, y, t)$ can be taken to be a local phase angle for the system, and from it all available information about the system can be obtained. We shall complete this section by showing how the total differential concept leads inevitably back to the Schrödinger equation with $\varphi$ the phase angle in the wave function.

The assumption that $d \alpha_{1}$ is the total differential and that the integral in (3.9) is to be taken along a dipolar centroidal path leads to the equation: 


$$
\frac{d \alpha_{1}}{d t}=\frac{\partial \alpha_{1}}{\partial t}+\frac{\partial \alpha_{1}}{\partial x} \frac{d x^{(c)}}{d t}+\frac{\partial \alpha_{1}}{\partial y} \frac{d y^{(c)}}{d t}=v\left(\tilde{\Omega}\left(r^{(c)}(t), t\right)+\Omega_{\mathrm{cx}}\left(r^{(c)}(t), t\right)\right)
$$

Or using (3.6) and (3.7), we get

$$
\partial \alpha_{1} / \partial t+\left(\partial \alpha_{1} / \partial x\right) u / 2+\left(\partial \alpha_{1} / \partial y\right) v / 2=v\left(k \cdot \nabla^{\wedge} \beta^{(c)}+\Omega_{\mathrm{ex}}\right)
$$

If we take the gradient through this equation, the result is

$$
\frac{\partial \nabla \alpha_{1}}{\partial t}+\beta^{(c)} \cdot \nabla \nabla \alpha_{1}+\left(\nabla \beta^{(c)}\right) \cdot \nabla \alpha_{1}=v \nabla\left(\widetilde{\Omega}+\Omega_{\mathrm{ex}}\right) .
$$

In the earlier work, we recognized that $\alpha_{1}$ is the velocity potential for the relative monopolar motion $\bar{\beta}$. However, if we recognize $\varphi$ as the local phase angle for the system, we can see that $\alpha_{1}$, which is equal to $v \varphi$, will play such a role for other reasons. Curves along which the phase angle $\varphi$ is constant are one-dimensional "synchronized" collections of moving elements and thus form an extended but united moving subsystem. The motion orthogonal to such equi-phase angle curves is in the direction of the "maximum" rate of change of the phase angle and consequently seems to be a "desynchronization" effect. On the other hand, motion along such a curve would seem to be a "synchronizationpreserving" effect. As sets of instantaneous monopole pairs or dipoles are the elements from which the synchronized subsystems are constructed, it seems that the route for the destruction of synchronized subsystems will lie along the direction of the relative monopolar motion $\bar{\beta}$. Consequently, the relative monopolar motion should be orthogonal to the equi- $\varphi$ curves. Thus $\alpha_{1}$ is the velocity potential for the relative monopolar motion. The vector version of this relation which was earlier expressed by (2.9) and (2.10) is

$$
\bar{\beta}=-\nabla \alpha_{1}
$$

We can reinforce the preceding remarks in terms of entropy or disorder considerations. If the direction along which phase synchronization occurs is regarded also as a direction of maximal ordering, and if maximal disorder is characterised by uniform or constant entropy, then along such a direction, the rate of change of entropy should be maximal. That is to say, the phase synchronization direction is orthogonal to the "entropic" 
equi-curves. It follows that the curves of equal entropy should be orthogonal to the curves of equal phase. Consequently, entropy should be given by the complex conjugate harmonic function to the harmonic function $\alpha_{1}$ That is, entropy is given by $\alpha_{2}$ in action units. It seems that from the present point of view (3.14) is a very well founded relationship. It has quite a spectacular result when substituted into equations (3.12) and (3.13) because it has the effect of making the second two terms in (3.13) equal; and, consequently, together they become $\beta \cdot \nabla \nabla \alpha_{1}$. The phase angle $\varphi$ and the velocity potential $\alpha_{1}$ for the relative monopolar flow are related by $\alpha_{1}=v \varphi$. Equation (3.12) becomes the Bernoulli equation for quantum flow (2.12) and equation (3.13) becomes the Newtonian equation for quantum flow,

$$
d p /\left.d t\right|_{\beta}=-m_{0} v \nabla\left(\tilde{\Omega}+\Omega_{e x}\right)
$$

where the differentiation in (3.15) now follows the monopolar velocity field $\beta$. The momentum $p$ is given by

$$
p=m_{0} \bar{\beta}(x, y, t)
$$

as the second component of the velocity field $\beta$ carries the negative mass $-m_{0}$ of the negative mass monopoles. It is clear from (3.15) that the usual external potential $V_{1}$ should be identified with the angular velocity component $m_{0} v k \cdot \Omega_{\mathrm{ex}}$ according to (3.2). The other contribution to the potential $m_{0} v \widetilde{\Omega}$ is the potential experienced by a particle as a result of "linear" feedback. That is, it is the extra potential to which a "quantum" particle is subjected in addition to the prescribed external field potential $V_{1}$

\section{SYSTEM ORIENTATION}

The source term $\Gamma^{(\mathrm{act})}(x, y, t)$ in the continuity equation for action (2.6), which is given in detail by equation (2.13), is an energy density which also gives the local time rate of "production" of action, as opposed to the action that flows about the $x-y$ plane in a "strictly" conserved sense. However, as the relation between action per dipole $\alpha_{1}$ and phase angle per dipole $\varphi$ is 


$$
\alpha_{1}=v \varphi,
$$

we can regard this source term as being a local source of phase angle "density". The idea of an "angular" density is not a "comfortable" concept. However, we shall use this idea in the following work as it does seem to have penetrating consequences in the analysis of this system. Using this idea which in this context is just a minor extension of the idea of action density (which itself is somewhat obscure), we see that the quantity in (2.15) that we are taking to be stationary or hopefully minimal, is in some sense a total angle through which the whole of the system within the region $S$ has rotated in the time interval $t-t_{0}$. Thus we define the change of orientation of that part of the system within the region $S$ and over the time interval $t-t_{0}$ as

$$
\begin{aligned}
\Delta \varphi^{(\mathrm{syst})} & =\left(v_{0} / m_{0} v S\right) \int_{t_{0}}^{t} \iint_{S} \Gamma^{(\mathrm{act})}\left(x, y, t^{\prime}\right) d x d y d t^{\prime} \\
& =\int_{t_{0}}^{t} \Omega^{\text {(syst) }} d t^{\prime} .
\end{aligned}
$$

The comparison volume $v_{0}$ has been introduced by

$$
\rho=\rho^{(0)} v_{0}^{-1},
$$

so that $\rho^{(0)}$ is the number of dipoles in the comparison volume $v_{0}$ which makes $\rho^{(0)} / v_{0}$ equal to the actual density.

$$
\Omega^{(\text {syst })}=1 / S \iint_{S} \Omega^{(\text {stir) }} d x d y
$$

and

$$
m_{0} c l_{0} \Omega^{(\mathrm{stir})}=E^{(\mathrm{stir})} \rho^{(0)}(x, y, t)
$$

In (4.6), the local angular velocity $\Omega^{\text {(stir) }}$ takes into account the additive property of "energy" in that there are $\rho^{(0)}$ dipoles within the volume $v_{0}$ each with energy $E^{(\text {stir) }} . \Omega^{\text {(syst) }}$ is clearly defined to be a subsystem average of $\Omega^{\text {(stir) }}$ locally for the subsystem contained within the region $S$, and it is a measure of the "rate" of rotation of the whole of this subsystem. It may be more appropriate to regard the angle $\Delta \varphi^{\text {(syst) }}$ not so much as a 
measure of the internal state of the subsystem enclosed within the region $S$, but rather as a measure of its relationship with its environment. This would perhaps be more obvious if the whole system could be enclosed in some finite region or if the integral over the whole hyperspace were convergent. However, it is not likely that either of these conditions would apply in most cases of physical interest. If we compare $\Delta \varphi^{(\text {syst })}$, the"system" angular orientation from (4.2), with the quantity $L^{\text {(syst) }}$, given in (2.16) that was used in the variational principle, we see that they only differ by a constant multiplier. Thus the variational principle that has been introduced can be expressed rather "loosely" as follows: "The internal state of a quantum system follows a path such that the disturbance of its overall angular orientation in the hyperspace is stationary."

\section{CONCLUSIONS}

It has been demonstrated that Schrödinger quantum theory can be derived from a very simple classical fluid variational principle, which involves our rendering stationary a quantity of clear physico-geometrical significance in the hyperspace in which this theory is couched. This result suggests that quantum systems evolve in such a way that the "disturbance" as measured by a system's rotation with respect to its environment is minimal. This is rather reminiscent of the idea in chemistry and biology that some moving systems evolve with minimum "rates" of entropy production in their environments [15]:

The general implication of the success of this type of approach in the study of quantum foundations is that the structure of orthodox quantum theory is a consequence of an underlying substratum of the more fundamental, "classical", fluid-like movement of a hypervacuum. This suggests strongly that orthodox quantum theory, at least as it is presently formulated and explained, should not be regarded as being a fundamental theory. This is a "philosophical" conclusion that certainly runs counter to the opinions of a majority within the scientific community. However, if the viewpoint of this article is seen to be nearer to the truth than is the orthodox view of the significance of the Schrödinger equation within the general study of quantum processes, then, at the very least, it would change the way the "paradoxical" features $[21,22]$ of the orthodox theory are seen and evaluated. At a more practical level, such a change in the assessment of the significance of orthodox quantum theory would help explaining the appearance of fluids with quantum-like characteristics under unexpected physical conditions. An obvious example is the appearance of superconductivity and superfluidity in materials at other than very low 
fundamental, more examples will come to light with time: and there will be no particular logic in explaining them on the basis of orthodox quantum theory.

\section{REFERENCES}

[1] Gilson, J.G., A Classical basis for the quantum mechanics, Ann. Inst. Henri Poincaré, XXXII nº 4, pp. 319-325, (1980).

[2] Gilson, J.G., The Nature of a Quantum State, Acta Physica Hungarica, 60 (3-4), pp. 145-160 (1986).

[3] Gilson, J.G., An Alternative View of the Schrödinger Process, Speculations in Science and Technology, Vol. 9 (1986).

[4] Gilson, J.G., Six-dimensional Flow and the Three-dimensional Schrödinger Equation, Journal of Mathematical and Physical Sciences, Vol 20, No. 1, pp. 55-63, (Feb. 1986).

[5] Gilson, J.G., A Real Plane Representation of the Electrodynamics of the Complex Schrödinger Configuration Plane, Annals de la Fondation Louis de Broglie, Vol 6, No. 1, (1981).

[6] Yourgraw, W., Variational Principles in Dynamics and Quantum Theory, Third ed., Pitman and Sons Ltd., London, (1968) .

[7] Cowing, T.G., Magnetohydrodynamics, Adam Hilger, (1976).

[8] Bullough, R.K., Caudry, P.G., Topics in Current Physics, Solitons, Springer-Verlag Berlin, (1980) .

[9] Rebbi, C., Soliani, G., Solitons and Particles, World Scientific (1984).

[10] Drazin, P.G., Solitons, Lecture Note Series 85, London Mathematical Society, Cambridge University Press. (1983). 
[11] Milne-Thomson, Theoretical Hydrodynamics, Macmillan and Co. Ltd., New York, (1949).

[12] Sommerfield, A., Thermodynamics and Statistical Mechanics, Vol. V, Academic Press, (1967).

[13] Landau and Lifshitz, Course of Theoretical Physics, Vol. 6, Pergamon Press, (1981).

[14] London, J., Superfluids, Vol. 11, John Wiley and Sons, Ltd., (1954).

[15] Prigogine, I., Thermodynamics of irreversible processes, Interscience, (1967).

[16] Madelung, E., Zs.f. Phys., 40, 332, (1926).

[17] Jánossy, L., Foundations of Physics, 6., 341, (1976).

[18] Takabayasi, T., Progress of Theor. Phys., 9, 187, (1953).

[19] De Broglie, L., La Thermodynamique de la Particle Isoleé, GauthierVillars, Paris, (1964).

[20] Jammer, M., The Philosophy of Quantum Mechanics, John Wiley and Sons, N.Y., (1974).

[21] Peres, A., Am. J. Phys., 54(8), August, (1986).

[22] Bohm, D., Hiley, B.J., Kaloyeru, P.N., An Ontological Basis for the Quantum Theory, Physics Reports, 144, No. 6, 323-349, North-Holland, Amersterdam, (1987).

[23] Barut, A.O., Class. Quantum Gravity, 4, 141, (1987).

[24] Barut, A.O., IC / $87 / 157$. 
[25] Cavalleri, G., LeTTERE AL NUOVO CIMENTO, Vol. 43, No. 6, 16 Luglio, (1985).

[26] Cavalleri, G., Spavieri G., IL NUOVO CIMENTO Vol. 95, B, No. 2, 11 Ottobre, (1986).

[27] Gilson, J.G., Classical Fluid Aspects of Nonlinear Schrödinger Equations and Solitons, Journal of Applied Mathematics and Simulation, Vol. 1, Issue 2, (1987). 


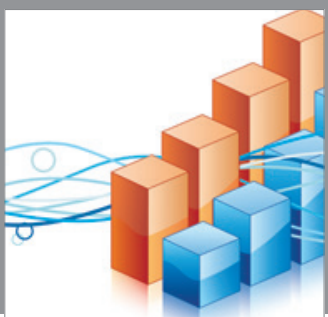

Advances in

Operations Research

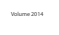

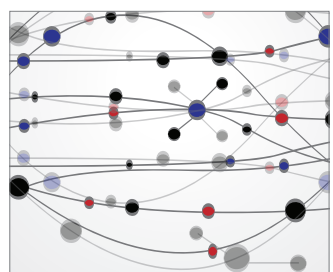

\section{The Scientific} World Journal
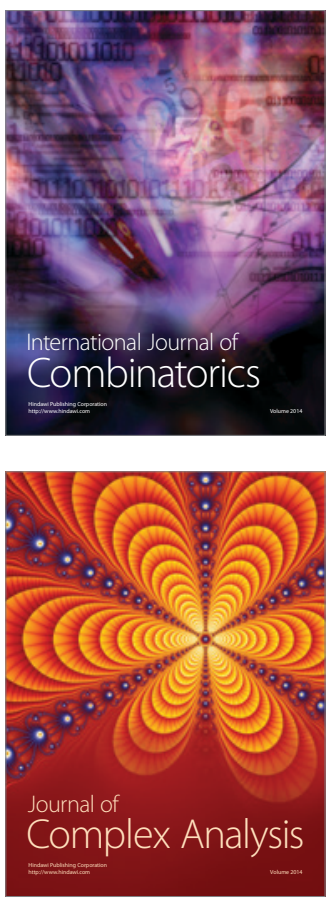

International Journal of

Mathematics and

Mathematical

Sciences
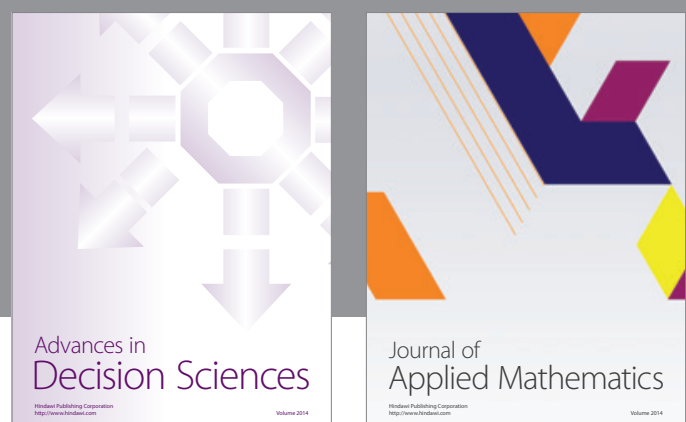

Journal of

Applied Mathematics
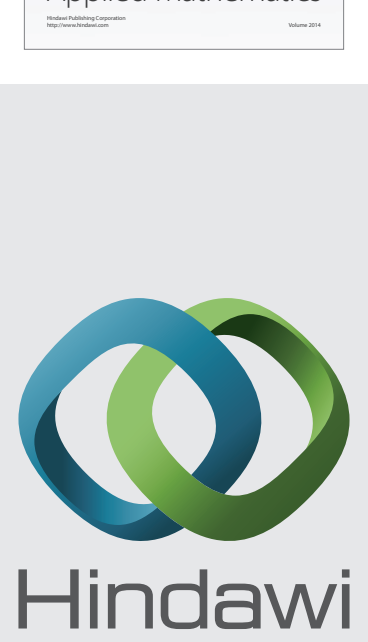

Submit your manuscripts at http://www.hindawi.com
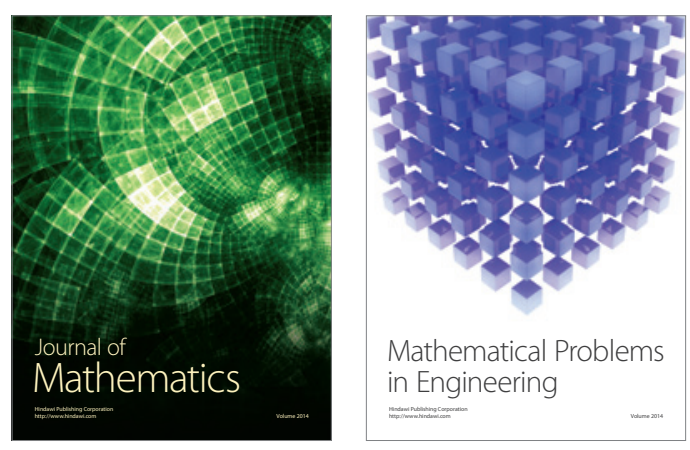

Mathematical Problems in Engineering
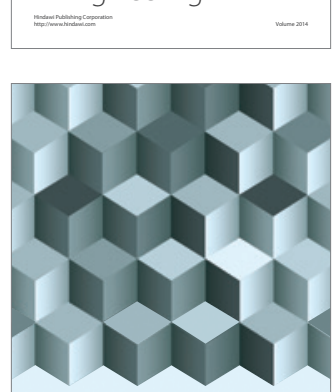

Journal of

Function Spaces
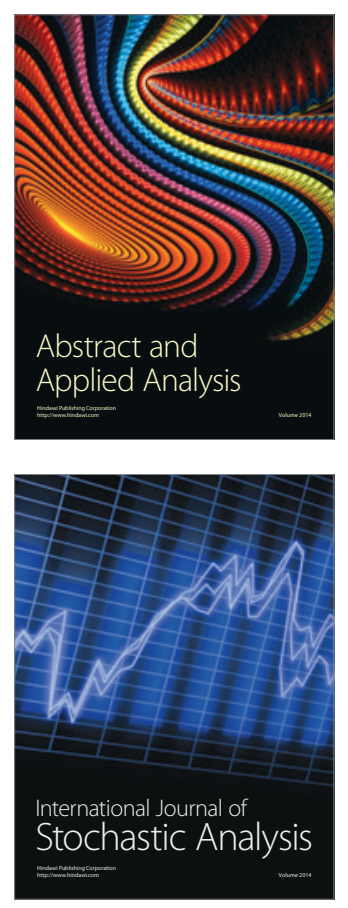

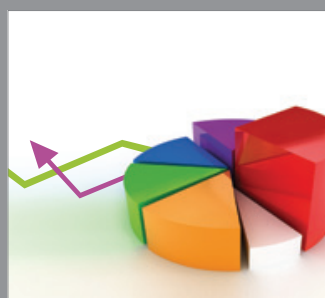

ournal of

Probability and Statistics

Promensencen
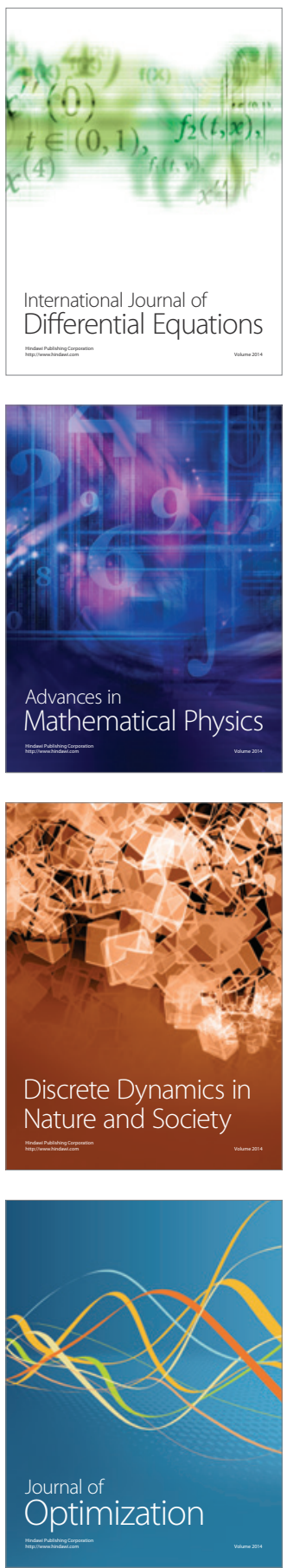